\title{
Jefferson.
}

The Medicine Forum

Volume 13

Article 8

2012

\section{An Indonesian Male with Abdominal Pain and Weight Loss: Abdominal Tuberculosis Presenting with Cervical Lymph Node Enlargement}

Leah Duke Kaye, MD

Thomas Jefferson University

Jayne Littlejohn, MD

Thomas Jefferson University

Follow this and additional works at: https://jdc.jefferson.edu/tmf

Part of the Medicine and Health Sciences Commons

Let us know how access to this document benefits you

\section{Recommended Citation}

Kaye, MD, Leah Duke and Littlejohn, MD, Jayne (2012) "An Indonesian Male with Abdominal Pain and Weight Loss: Abdominal Tuberculosis Presenting with Cervical Lymph Node Enlargement," The Medicine Forum: Vol. 13 , Article 8.

DOI: https://doi.org/10.29046/TMF.013.1.009

Available at: https://jdc.jefferson.edu/tmf/vol13/iss1/8

This Article is brought to you for free and open access by the Jefferson Digital Commons. The Jefferson Digital Commons is a service of Thomas Jefferson University's Center for Teaching and Learning (CTL). The Commons is a showcase for Jefferson books and journals, peer-reviewed scholarly publications, unique historical collections from the University archives, and teaching tools. The Jefferson Digital Commons allows researchers and interested readers anywhere in the world to learn about and keep up to date with Jefferson scholarship. This article has been accepted for inclusion in The Medicine Forum by an authorized administrator of the Jefferson Digital Commons. For more information, please contact: JeffersonDigitalCommons@jefferson.edu. 


\title{
An Indonesian Male With Abdominal Pain and Weight Loss: Abdominal Tuberculosis Presenting With Cervical LYMPH NODE ENLARGEMENT
}

\author{
Leah Duke Kaye, MD and Jayne Littlejohn, MD
}

\section{Case}

A 21-year-old Indonesian male presented with a 6-month history of abdominal pain. The pain was described as a diffuse cramping that was worse with movement and after meals. He reported early satiety, and the pain was improved at rest. Associated with the pain, he reported a seventy pound weight loss, chills, decreased energy, and fatigue over the same time period. He denied nausea, vomiting, fevers, cough, dyspnea, or chest pain.

The patient emigrated from Indonesia to the United States in 2007 and has since had no travel or foreign visitors. He denied incarceration or military duty for both himself and close relatives. There was no prior purified protein derivative (PPD) placement or Bacille Calmette Guerin (BCG) vaccination.

The patient was seen at a different hospital approximately one month prior for a subcutaneous mass in his right lateral neck that had been present for two years. At that time, the mass was diagnosed as a simple cyst on imaging; no fluid aspiration or biopsy was performed. During the month that elapsed before presentation to our hospital, the patient reported the mass spontaneously drained yellow fluid and dramatically reduced in size.

On examination, he was afebrile and in no distress, but he appeared cachectic and older than his stated age. There was a right supraclavicular indurated healed scar without fluctuance or discharge. The patient had clear lungs to auscultation. On abdominal exam, he had loose skin with pale striae, normal bowel sounds, and a soft non-distended abdomen with tenderness to palpitation in the lower quadrants. There was mild guarding, but no rebound, hepatosplenomegaly, or bulging flanks.

Initial blood work was significant for a normocytic anemia, with a hemoglobin of $12.4 \mathrm{~g} / \mathrm{dL}$, a mean cell volume of $81 \mathrm{fL}$, platelet count of $410 \mathrm{~B} / \mathrm{L}$, and a mildly elevated white blood cell count of $12.4 \mathrm{~B} / \mathrm{L}$. All other routine chemistries including measures of renal and hepatic function were within normal limits.

A computed tomography (CT) of the abdomen and pelvis revealed a small amount of intra-abdominal ascites with numerous ring-enhancing lesions seen throughout the peritoneum along the colon, particularly the sigmoid, and along the serosal surface of the liver. There were numerous enlarged mesenteric and retroperitoneal lymph nodes as well as enlarged right and left pericardiophrenic lymph nodes (Figure 1).

During his hospital course, a PPD was placed and was positive with a $15 \times 10 \mathrm{~mm}$ area of induration. Sputum, blood, stool, and urine cultures were sent for acid fast bacilli (AFB) and were all negative on smear. The patient's prior CT scan of his neck and

thorax was obtained from the outside hospital. Our radiology department interpreted what was called a simple cyst at the outside hospital as a necrotic lymph node in the right lateral aspect of the neck extending from the C5-C6 level through the supraclavicular fossa (Figure 2), and the CT thorax was notable for trace left pleural effusion without adenopathy or cavitary lesions. The patient underwent an ultrasound guided fluid aspiration of the cervical lymph node.

An exploratory abdominal laparoscopy was performed for confirmatory diagnosis of peritoneal tuberculosis. The procedure revealed peritoneal studding, and multiple nodules

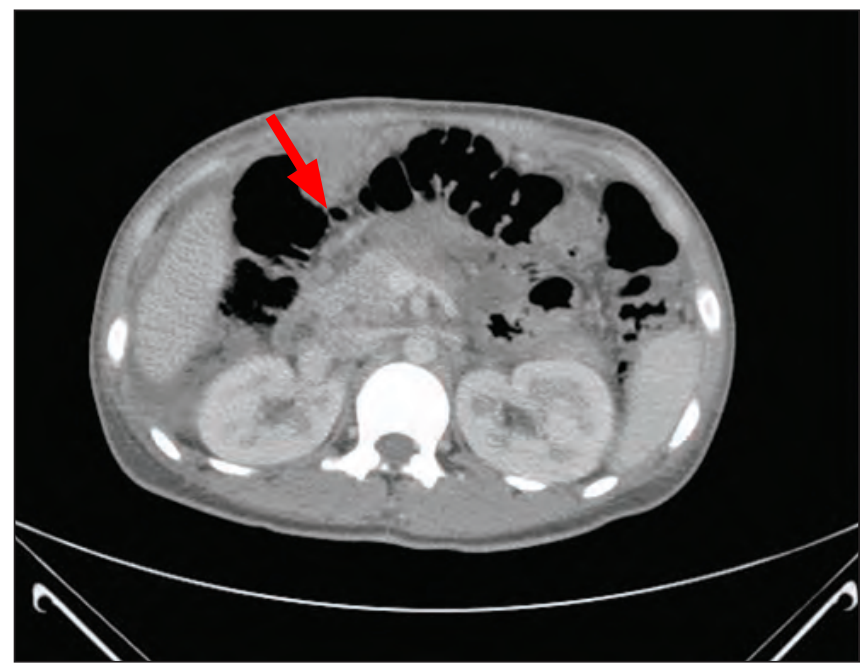

Figure 1. Ring-enhancing lesion on the serosal surface of the distal transverse colon measuring $2.4 \times 2.1 \mathrm{~cm}$ (red arrow)

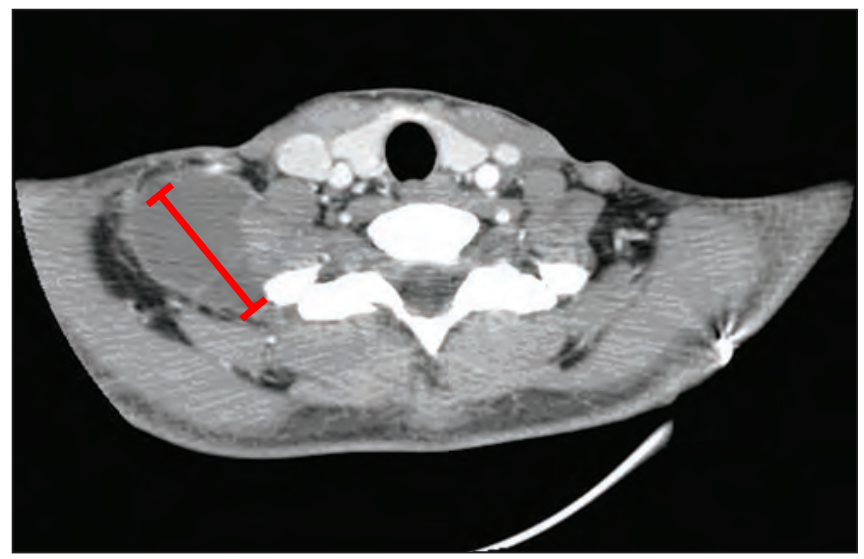

Figure 2. $39.1 \mathrm{~mm} X 46.7 \mathrm{~mm}$ mass (red arrow) along the right lateral aspect of the neck extending from the C5-C6 level through the supraclavicular fossa 
were noted on the anterior abdominal wall. Biopsy of the lesions revealed a caseous exudate in the operating room. Peritoneal fluid was not obtained due to the minimal ascites. Surgical pathology reported necrotizing granulomas, and an acid-fast stain identified AFB. The AFB culture sent from the OR was positive for Mycobacterium tuberculosis complex thirty-six days later. The organism was susceptible to isoniazid, streptomycin, ethambutol, and rifampin. The aspirate of the cervical lymph node also grew $M$. tuberculosis with the same sensitivities as the abdominal culture.

The patient was empirically started on a regimen of ethambutol $1200 \mathrm{mgs}$ daily, isoniazid $300 \mathrm{mgs}$ daily, pyrazinamide $1500 \mathrm{mgs}$ daily, pyridoxine $50 \mathrm{mgs}$ daily, and rifampin $600 \mathrm{mgs}$ daily. $\mathrm{He}$ was discharged on hospital day 11 to continue directly observed therapy as an outpatient with the Department of Public Health.

\section{Discussion}

Approximately one-fifth of tuberculosis (TB) cases in the United States are purely extra-pulmonary. Of these, approximately $4.9 \%$ are peritoneal. The odds of presenting with extrapulmonary tuberculosis versus pulmonary tuberculosis are over 15 times higher for females, Asians, foreign-born residents, and healthcare workers, with $81 \%$ of cases seen in nonwhite racial/ethnic groups and $15 \%$ of cases seen in patients born in Southeast Asia. Unlike pulmonary or disseminated TB, there are not strong associations between extra-pulmonary tuberculosis and age, homelessness, excess alcohol use, or HIV status. Both extra-pulmonary and pulmonary tuberculosis cases have decreased in the U.S., but extra-pulmonary cases have decreased less-leading to an increase in extra-pulmonary tuberculosis as a proportion of total cases. In 1962, only $7.6 \%$ of tuberculosis cases were extra-pulmonary; in 2006 that number had increased to $21 \%{ }^{1}$

The common presenting symptoms of abdominal TB are anorexia, fever, weight loss, and abdominal pain/tenderness. ${ }^{2,3,4}$ More than $70 \%$ of patients have symptoms for more than four months before their diagnosis is actually established. ${ }^{5,6}$ The most common finding on physical exam is ascites. ${ }^{7}$ Other common findings include an abdominal mass, "doughy" abdomen, and hepatomegaly. ${ }^{2,4}$ Routine labs are generally unremarkable except for a mild to moderate normocytic, normochromic anemia. ${ }^{4}$

CT of the abdomen, while not necessarily diagnostic, can be extremely helpful in suggesting the diagnosis of abdominal tuberculosis. Ascites, peritoneal lesions, and lymphadenopathy are common findings seen in these patients. ${ }^{8}$ More specific features are free ascites, thickened strands with crowded vascular bundles within the mesentery, smudged pattern of omental involvement, and smooth, uniform thickening of the peritoneum. ${ }^{10}$ The commonest form of extrapulmonary tuberculosis is scrofula, also known as cervical lymphadenitis. ${ }^{9}$

A number of tests are available to attempt to make the diagnosis of abdominal tuberculosis, including tuberculin skin testing, ascitic fluid analysis, ascitic culture, and ascitic adenosine deaminase levels. A PPD is positive in approximately $70 \%$ of patients, but a negative test cannot exclude the diagnosis. ${ }^{11}$ Peritoneal tuberculosis can be considered in any patient with unexplained ascites with a serum albumin-ascites gradient (SAAG) less than $1.1 \mathrm{~g} / \mathrm{dL}$. More than $95 \%$ of patients have a protein content over $3.0 \mathrm{mg} / \mathrm{dL}^{12}$ An attempt should be made to perform an acid-fast smear of ascitic fluid, as well as ascitic culture. However, diagnostic yield for acid-fast bacilli seen on smear is reported to be between $0-6 \%{ }^{4,13}$ and a positive culture is achieved in less than $20 \%$ of cases. ${ }^{10}$ Moreover, culture can take up to 6-8 weeks to grow, making it a less attractive option. Another approach to ascitic fluid involves the measurement of adenosine deaminase (ADA) levels, an enzyme necessary for differentiation and growth of T cells. A level of ascitic ADA over $39 \mathrm{IU} / \mathrm{L}$ has a $100 \%$ sensitivity and $97 \%$ specificity in patients without cirrhosis. ${ }^{14}$

If the above tests are insufficient to diagnose tuberculous peritonitis, exploratory laparoscopy or laparatomy, preferably with peritoneal biopsy, becomes the next step. A visual diagnosis upon seeing visceral and parietal peritoneum studded with multiple whitish nodules, enlarged lymph nodes, "violin-string" fibrinous strands, and omental thickening can be diagnostic in $95 \%$ of cases. ${ }^{7,15}$ Upon visualizing lesions, a targeted biopsy is positive for acid-fast bacilli in up to $74 \%$ of patients and reveals caseating granulomas in up to $100 \%$ of patients. ${ }^{11,16}$

Initial treatment involves the same approach as pulmonary tuberculosis, with at least a 4-drug regimen until sensitivities can be determined. Conventionally, therapy involves rifampin, isoniazid, pyrazinamide, and ethambutol for at least 2 months, and therapy that continues for 6 months total that may use less drugs if sensitivities allow. Treatment may continue in some cases for 12-18 months. ${ }^{12}$ Fever generally resolves within one week of the start of anti-tuberculosis treatment; over $90 \%$ of patients have improvement of their ascites in a few weeks. ${ }^{17}$ Some research suggests the addition of methylprednisolone therapy for one month leads to earlier improvement of ascites and abdominal pain as compared to patients who do not receive the steroid. ${ }^{17}$ Prednisone treatment also been suggested for 1-3 months during the initial treatment as a means of reducing late complications from adhesive disease, such as chronic abdominal pain and bowel obstruction. ${ }^{18}$

Surgical approach to treatment was more popular before antitubercular drugs were available, often involving a right hemicolectomy due to frequent involvement of the ileocecal valve. However, with the development of available pharmacotherapy, this approach has generally fallen out of favor. If the patient develops strictures from the disease, stricturoplasty is an option; if there are a very high number of strictures, resection may be performed. ${ }^{12}$ However, even in the case of strictures, some reports suggest up to $70 \%$ of strictures may completely resolve with conventional antituberculosis therapy alone. ${ }^{19}$ In light of these reports, even patients with strictures should likely 
be given a trial of anti-tubercular therapy before surgery is considered.

The outcome of treated abdominal tuberculosis appears to depend heavily on the time between symptom onset and treatment. There are reports of up to $60 \%$ mortality rate if treatment is not started within 30 days of presentation, perhaps due to excessively advanced disease. ${ }^{4}$ The presence of cirrhosis is also an important prognostic factor. In one study, $16 \%$ of non-cirrhotics versus $48 \%$ of cirrhotics were not diagnosed with abdominal tuberculosis until death. ${ }^{4}$ This may be due to increased difficulty of diagnosis in cirrhosis, and underscores the importance of maintaining a high index of suspicion for abdominal tuberculosis.

\section{References}

1. Peto, Heather M., Pratt, Robert H, Harrington, Theresa A., LoBue, Philip A, and Armstrong, Lori R. Epidemiology of Extrapulmonary Tuberculosis in the United States, 1993-2006. Clinical Infectious Disease 2009; 49:1350-7

2. Chaudery, Muzzafer, Mohamed, Faheez, Shirol, Sunil. An unusual presentation of intraabdominal tuberculosis in a young man. Journal of the Royal Society of Medicine, 2010; 103: 199-201.

3. al-Quorain, AA, Facharzt, Satti, MB, et al. Abdominal tuberculosis in Saudi Arabia: A clnicopathological study of 65 cases. Am J Gasroenterology 1993; $88: 75$

4. Chow, KM, Chow, VC, Hung, LC, et al. Tuberculous peritonitis-associated mortality is high among patients waiting for the results of mycobacterial cultures of ascitic fluid samples. Clinical Infectious Diseases 2002 Aug 15;35(4):409-13. Epub 2002 Jul 17.

5. Lisehora, GB, Peters, CC, Lee, YT, Barcia, PJ. Tuberculous peritonitis-do not miss it. Dis Colon Rectum 1996; 39:394.
6. Gitt, S, Haddad, F, Levenson, S. Tuberculous peritonitis: an overlooked diagnosis. Hosp Pract (Off Ed) 1992; 27:224.

7. Bhargava, DK, Shriniwas, Chopra, P, et al, Peritoneal tuberculosis: laparoscopic patterns and its diagnostic accuracy. Am J Gastroenterol 1992; 87: 109

8. Vaquez Munoz, E, Gomez-Cerezo, J, Atienza Saura, M, Vazquez Rodriguez, JJ. Computed tomography findings of peritoneal tuberculosis: systematic review of seven patients diagnosed in 6 years (1996-2001). Clin Imaging 2004; 28: 340.

9. R Khan, S H Harris, A K Verma, A Syed. Cervical Lymphadenopathy: Scrofula Revisited. The Journal of Laryngology \& Otology (2009), 123: 764-767

10. Na-ChiangMai W, Pojchamarnwiputh S, Lertprasertsuke N, Chitaparanux T. CT findings of tuberculous peritonitis. Singapore Medical Journal 2008; 49(6): 488

11. Marshall, John. Tuberculosis of the gastrointestinal tract and peritoneum. Am J Gasroenterology 1993; 88:989

12. Manohar, A, Simjee, AE, Haffejee, AA, Pettengell, KE. Symptoms and investigative findings in 145 patients with tuberculous peritonitis diagnosed by peritoneoscopy and biopsy over a five year period. Gut 1990; 31: 1130

13. Sharma MP, Bhatia V. Abdominal tuberculosis. Indian Journal of Medical Research, 2004; 120:305-15

14. Riquelme, A, Calvo, M, Salect, F, et al. Value of adenosine demainase (ADA) in ascitic fluid for the diagnosis of tuberculous peritonitis: a meta-analysis. J Clin Gastroenterol 2006; 40: 705.

15. Uzunkoy, A, Harma, M, Harma, M. Diagnosis of abdominal tuberculosis: experience from 11 cases and a review of the literature. World J Gastroenterol 2004; 10:3647.

16. Chow, KM, Chow, VC, Szeto, CC. Indication for peritoneal biopsy in tuberculous peritonitis. Americal Journal of Surgery 2003; 185: 567.

17. Demir, K, Okten, A, Kaymakoglu, S, et al. Tuberculous peritonitis-reports of 26 cases, detailing diagnostic and therapeutic problems. Eur J Gastroenterol Hepatol 2001; 13: 581.

18. Alrajhi AA, Halim MA, al-Hokail A, et al. Corticosteroid treatment of peritoneal tuberculosis. Clin Infect Dis 1998;27:52-56.

19. Anand BS, Nanda R, Sachdev GK. Response of tuberculous stricture to antituberculous treatment. Gut 1988; $29: 62-9$.

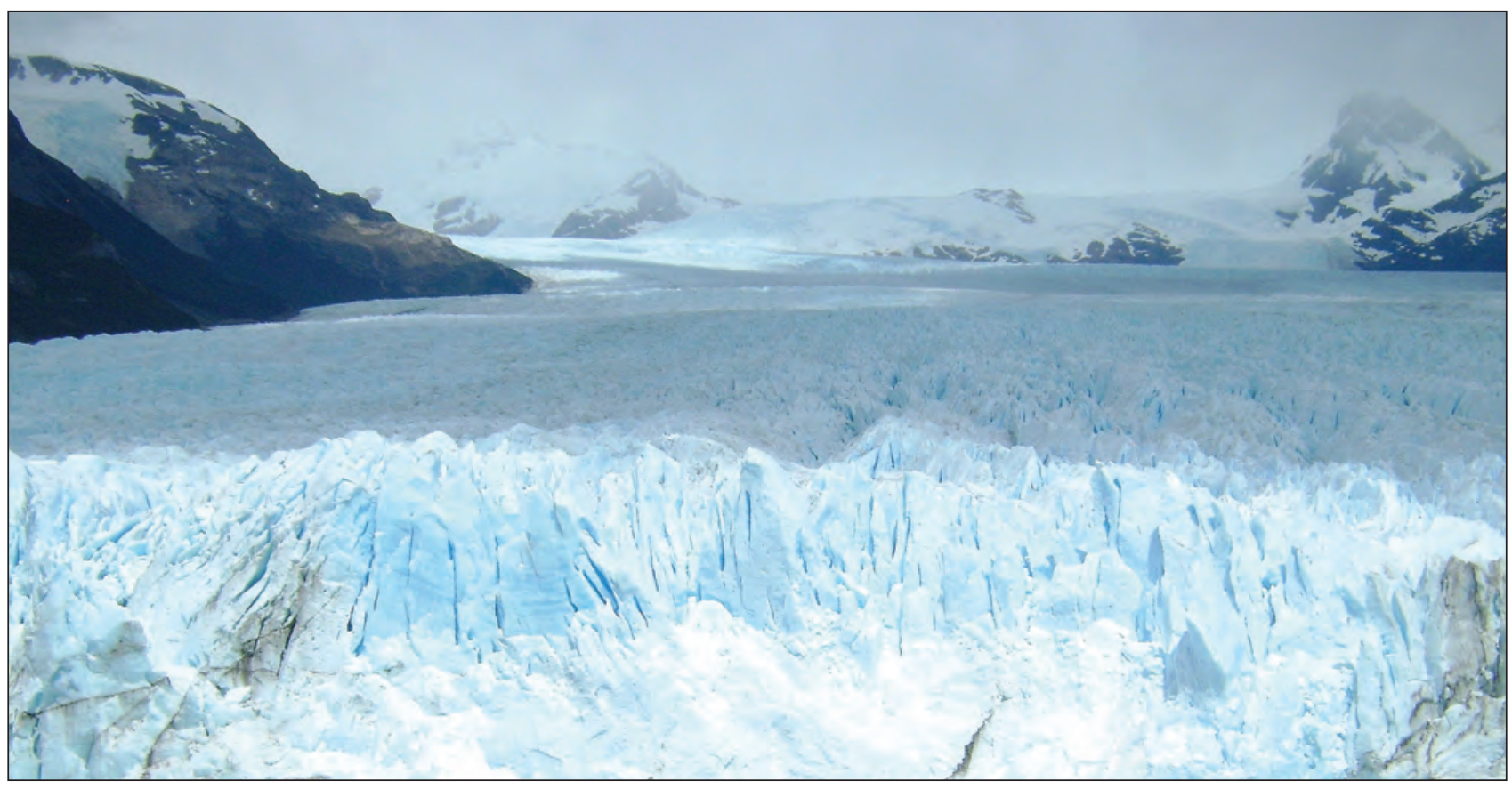

